\title{
Numerical and Experimental Investigation of Newtonian Flow around a Confined Square Cylinder
}

\author{
Guler Bengusu Tezel ${ }^{1 *}$, Kerim Yapici², Yusuf Uludag³ \\ 1 Department of Chemical Engineering, Faculty of Engineering and Architecture, Abant Izzet Baysal University, \\ 14280, Bolu, Turkey \\ 2 Department of Chemical Engineering, Faculty of Engineering and Architecture, Suleyman Demirel University, \\ 32260, Isparta, Turkey \\ ${ }^{3}$ Department of Chemical Engineering, Faculty of Engineering, Middle East Technical University, 06800 Ankara, Turkey \\ * Corresponding author, e-mail: bengusutezel@gmail.com.tr
}

Received: 09 April 2018, Accepted: 25 July 2018, Published online: 29 August 2018

\begin{abstract}
The confined flow of a Newtonian fluid around a square cylinder mounted in a rectangular channel was investigated both numerically and experimentally. Ratio between the pipe and channel height, the blockage ratio, is kept constant at 1/4. The flow variables including streamlines, vorticity and drag coefficients were calculated at $0 \leq R e \leq 50$ using finite volume method. The velocity terms in the momentum equations are approximated by a higher-order and bounded scheme of Convergent and Universally Bounded Interpolation Scheme for the Treatment of Advection (CUBISTA). Particle Image Velocimetry (PIV) was also used to obtain the twodimensional velocity field. The flow measurements were conducted for $1 \leq R e \leq 50$. Streamline and vorticity results obtained by PIV are compared with those of the numerical simulation. Based on this comparison, good agreement is found between the numerical and experimental results in a qualitative manner.
\end{abstract}

Keywords

square cylinder, confined channel, newtonian fluid, finite volume method, PIV

\section{Introduction}

Numerical analysis of the flow past over bluff bodies have been conducted in fluid mechanical studies for a long time [1-4]. The analysis of external flow past a square cylinder is used various engineering applications such as shell and tube heat exchangers [3-7], coating processes, cooling towers, extruders and membrane processes. It involves complex phenomena like flow separation and reattachment, drag formation. Most experimental and numerical studies [1-4] on the flow past a stationary square cylinder have been performed at moderate to high Reynolds numbers. In this flow is unsteady. There are also many steady flow studies on square cylinders.

Characteristics of the steady confined flow past a square cylinder have been studied by Breuer et al. [8]. They presented results for $R e=0.5-300$ in two-dimensional flow. The results were computed using finite-volume and lattice-Boltzmann simulations with a blockage of $1 / 8$. Separation was not observed for $R e<1$. Gupta et al. [9] studied the steady flow and heat transfer characteristics in relation with the power-law fluids for $R e=5-40$ and $B=1 / 8$. Sharma and Eswaran [10] presented results for $B=1 / 20$ and $R e=$ $1-160$ by using a finite-volume formulation. These studies are related to the physics of the Newtonian flow past a square cylinder and the accuracy of numerical predictions of simulations. In recent study, Rashidi et al. [3] studied the flow and heat transfer in a multiple square obstacles using finite volume method at constant $R e=100$. They observed that heat transfer enhances as the space ratio enhances between obstacles due to causing higher temperature gradient around channel wall and obstacles.

The results of the theoretical studies (especially numerical simulations) on fluid flow mechanics should be evaluated with respect to the experimental studies performed at similar conditions. In this study, Particle Image Velocimetry (PIV) is used to carry out flow field measurements experimentally. PIV has been used for both Newtonian and non-Newtonian fluid flow measurements [11]. This technique enables the qualitative and 
quantitative flow visualization by means of accurate measurement at multiple points over the entire flow.

In this study, Newtonian flow around square cylinder with $B=1 / 4$ is analyzed by developing non-uniform staggered 372 x 162 grids on finite volume code as in Fig. 1. Objective of this study is twofold. One is to obtain accurate numerical solutions of the system providing flow physics simulations. The other one is to compare and verify to the corresponding streamline and vorticity profiles using PIV measurements.

\section{Governing equations and numerical methodology}

In this study steady incompressible and isothermal flow of a Newtonian fluid over a 2D confined square cylinder is considered. The flow system is schematically depicted in Fig. 2. The blockage ratio, defined as the ratio between heights of the square obstacle and the channel is $1 / 4(\mathrm{~b} / \mathrm{H}=1 / 4)$. The length of channel is set as $\mathrm{L} / \mathrm{H}=30$ while the downstream channel length is $1 / 24$ to ensure fully developed flow.

The formulation of the flow begins by considering basic equations of fluid flow, i.e. dimensionless form of the continuity and momentum equations (Eqs. (1)-(3)) given below [12-14].

Continuity

$$
\frac{\partial u}{\partial x}+\frac{\partial v}{\partial y}=0
$$

$\mathrm{x}$-momentum

$$
\begin{gathered}
\frac{\partial u u}{\partial x}+\frac{\partial v u}{\partial y}=-\frac{\partial p}{\partial x}+\frac{1}{\operatorname{Re}}\left(\frac{\partial^{2} u}{\partial x^{2}}+\frac{\partial^{2} u}{\partial y^{2}}\right) \\
\text { y-momentum } \\
\frac{\partial u v}{\partial x}+\frac{\partial v v}{\partial y}=-\frac{\partial p}{\partial y}+\frac{1}{\operatorname{Re}}\left(\frac{\partial^{2} v}{\partial x^{2}}+\frac{\partial^{2} v}{\partial y^{2}}\right) .
\end{gathered}
$$

A finite volume method [13, 14] with non-uniform staggered grid is used to obtain discrete form of the flow equations. Second order central difference scheme is used for the approximation of the diffusion terms in the momentum equations. Convective terms in the equations are approximated by at least second-order accurate, bounded and non-uniform version of CUBISTA [13] scheme given in Eq. (4). The implementation of the CUBISTA scheme is carried out via deferred correction method that was proposed by Rubin and Khosla [16]. Hayase et al. [17] reported a study by for the formulation of uniform QUICK scheme into CUBISTA scheme. This scheme is preferred due to its

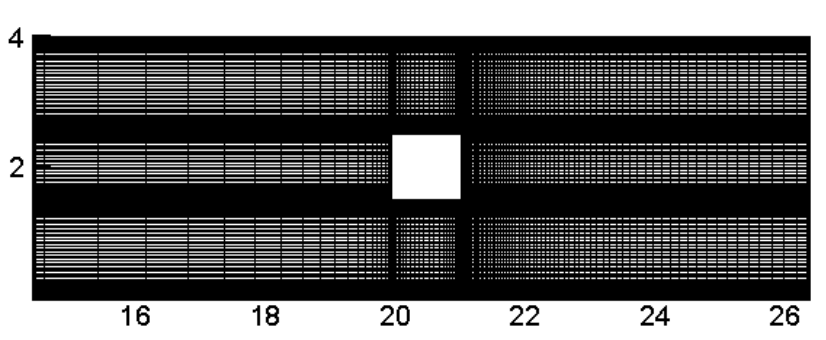

Fig. 1 Non-uniform mesh around the obstacle with minimum cell size of $\Delta \mathrm{x}=0.02$ and $\Delta \mathrm{y}=0.01$ for $\mathrm{B}=1 / 4$

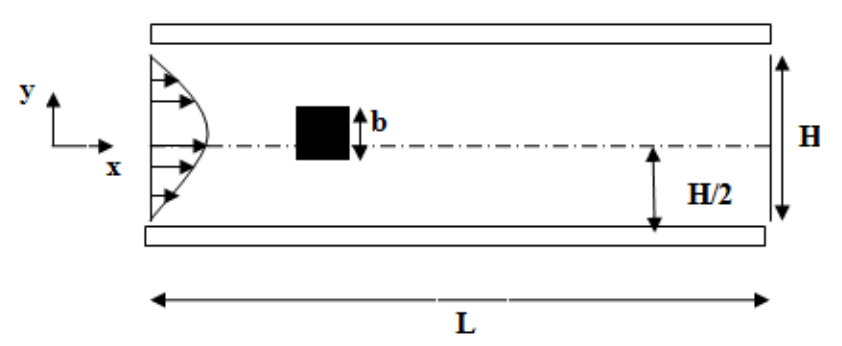

Fig. 2 Schematic of 2D flow around a square cylinder

documented advantages on the higher order schemes [16]. The purpose of the convection scheme use is then to specify the values of $\phi_{f}\left(\phi_{w}, \phi_{e}\right)$ at the face, based on existing values at the neighbouring cell centres $\phi_{c}\left(\phi_{P}\right)$ with their locations as $x_{W}, x_{w}, x_{P}, x_{e}, x_{E}$ in Fig. 3. The simplest scheme satisfying the transportive property is upwind, whereby $\phi_{f}=\phi_{P}$ where $P$ is the cell centre situated on the upwind side in relation to face $f$ (measured by $F_{f}>0$ ). With using coordinates notations in Fig. 3, generalized form of CUBISTA is given as:

$$
\hat{\phi}_{f}=\left\{\begin{array}{ll}
\left(1+\frac{\hat{\xi}_{f}-\hat{\xi}_{P}}{3\left(1-\hat{\xi}_{P}\right)}\right) \frac{\hat{\xi}_{f}}{\hat{\xi}_{P}} \hat{\phi}_{P} & 0<\hat{\phi}_{P}<\frac{3}{4} \hat{\xi}_{P} \\
\frac{\hat{\xi}_{f}\left(1-\hat{\xi}_{f}\right)}{\hat{\xi}_{P}\left(1-\hat{\xi}_{P}\right)} \hat{\phi}_{P}+\frac{\hat{\xi}_{f}\left(\hat{\xi}_{f}-\hat{\xi}_{P}\right)}{\left(1-\hat{\xi}_{P}\right)} & \frac{3}{4} \hat{\xi}_{P} \leq \hat{\phi}_{P} \leq \frac{1+2\left(\hat{\xi}_{f}-\hat{\xi}_{P}\right)}{2 \hat{\xi}_{f}-\hat{\xi}_{P}} \hat{\xi}_{P} \\
1-\frac{\left(1-\hat{\xi}_{f}\right)}{2\left(1-\hat{\xi}_{P}\right)}\left(1-\hat{\phi}_{P}\right) & \frac{1+2\left(\hat{\xi}_{f}-\hat{\xi}_{P}\right)}{2 \hat{\xi}_{f}-\hat{\xi}_{P}} \hat{\xi}_{P}<\hat{\phi}_{P} \\
\hat{\phi}_{P} & \text { elsewhere }
\end{array} .\right.
$$

Where

$\hat{\phi}_{f}=\frac{\phi_{f}-\phi_{E}}{\phi_{E}-\phi_{W}} \quad \hat{\xi}_{P}=\frac{x_{P}-x_{E}}{x_{E}-x_{W}} \quad$ and $\quad \hat{\xi}_{f}=\frac{x_{e}-x_{E}}{x_{E}-x_{W}}$.

The final form of the two dimensional discretized governing equations over a control volume can be expressed symbolically as follows using finite volume method:

$A_{P} \phi_{i, j}=A_{E} \phi_{i+1, j}+A_{W} \phi_{i-1, j}+A_{N} \phi_{i, j+1}+A_{S} \phi_{i, j-1}+b$

where the coefficients are approximated by the upwind differencing scheme. The SIMPLE [17] method is employed 


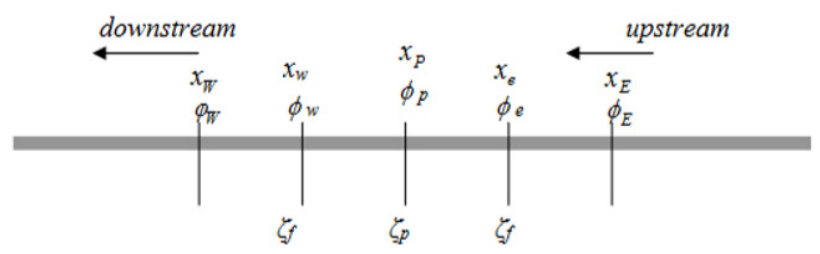

Fig. 3 General representation for grid points in the $\mathrm{x}$ direction one dimensional Cubista

to handle the coupled system of the continuity, momentum and equations. The set of linearized algebraic equations are then solved by using the Thomas algorithm or the tridiagonal matrix algorithm (TDMA). The solution process is reiterated until the maximum relative change of flow variables, $\varnothing_{i, j}(u, v, p)$ is less than a prescribed tolerance or residual as:

$r e s=\operatorname{MAX}\left\{\frac{\left|\phi^{n+1}-\phi^{n}\right|}{\left|\phi^{n+1}\right|}\right\} \leq 1 \times 10^{-8}$.

Use of correct boundary conditions especially for stress components is crucial to capture the physics of the flow. The following inlet conditions are imposed for $x$ and $y$-components of the velocity, $u$ and $v$ respectively.

Inlet Boundary Conditions:

$u=1-\left|(1-0.5 y)^{2}\right|$

$v=0$

$0 \leq y \leq 4$.

No slip boundary conditions are assumed at the solid-fluid interfaces:

$u=0$

$v=0$.

At the channel exit Neumann boundary conditions are imposed for the flow variables:

$\frac{\partial u}{\partial x}=0, \frac{\partial v}{\partial x}=0, \frac{\partial P}{\partial x}=0$.

\section{Numerical Results and Discussion}

Fig. 4 shows the effect of Re number on the confined flow patterns around the square obstacle in terms of streamline and vorticity profiles for $R e=0,10,20,30,50$. The streamline profiles are shown in the upper half of figures, while the vorticity contours are shown in the lower half. No separation occurs from the surface of the cylinder for $R e=0$ due to creeping nature of the flow. However, flow separation is observed at higher Re numbers. Near the cylinder the streamline and vorticity profiles are quite similar but there are growing size differences further downstream, indicating the greater development of the wake as the Reynolds number increases from 0 to 50 . The length and size of the vortex obtained also increased with growing wake region as in the Fig. 4.

The flow separation gets more pronounced at the vicinity of the obstacle edges as increased $R e$ number. A closed recirculation region consisting of two symmetric vortices develop in the wake region as shown in Figs. 4(c)-(e). Dimensionless recirculation length that is also known as the wake region is defined by Breuer et al. [8] as the distance between the obstacle surface and reattachment point of streamlines (i.e., $\psi=0$ on the axis of symmetry at $y=2$ ) to form the encapsulated region behind the obstacle. As Reynolds increased, this length also gets larger.

Another important flow characteristic is vorticity distribution around the obstacle. Vorticity profiles can also be used to investigate the behavior of the fluid flow around the obstacle. Stream function, $\psi$, and vorticity, $\omega$, are obtained through the solution of the following Eqs. (10)-(11).

$\nabla^{2} \psi=-\omega$

$\omega=-\frac{\partial u}{\partial y}+\frac{\partial v}{\partial x}$

Vorticity are also helpful in locating separation points. These contours seem to transit from being symmetrical at $R e=0$ to being asymmetrical at higher $R e$ numbers as in Fig. 4. The magnitude of the corner vorticity (at $x=20$ ) increases with $R e$ number at the upstream of the flow for $R e=20,30,50$. To do detail analysis of vorticity, some primary points are chosen. Values of the stream function and vorticity and their primary axial wake locations at $x=22,23,24$ and vertical locations at $y=2.5,3$ are tabulated in Table 1. These points are also considered as near wake $(x=22, y=2.5)$ and far wake region $(x=24$, $y=3$ ) of the obstacle. In the near wake region, vorticity values $(\omega=-4.3842$ at $R e=0)$ are larger compared to far wake region vorticity $(\omega=-1.4988$ at $R e=0)$ due to highly intense velocity gradients in that region. Gupta et al. [9], Dhiman et al. [10], Sharma and Eswaran [25] have also reported similar vorticity distribution for Newtonian flow around the square cylinder in the range conditions $1 \leq$ $R e \leq 45$. For higher $R e$ flows, vicinitiy vorticities (at $x=20$, $y=1.5)$ get higher in Figs. 4(c)-(e), since $v$-velocity component gradient is also higher at small $R e$ as seen in Fig. 5.

For the same region, say $x=22$ and $y=2.5$, streamline magnitudes are similar for different $R e$ number as in Table 1 . 

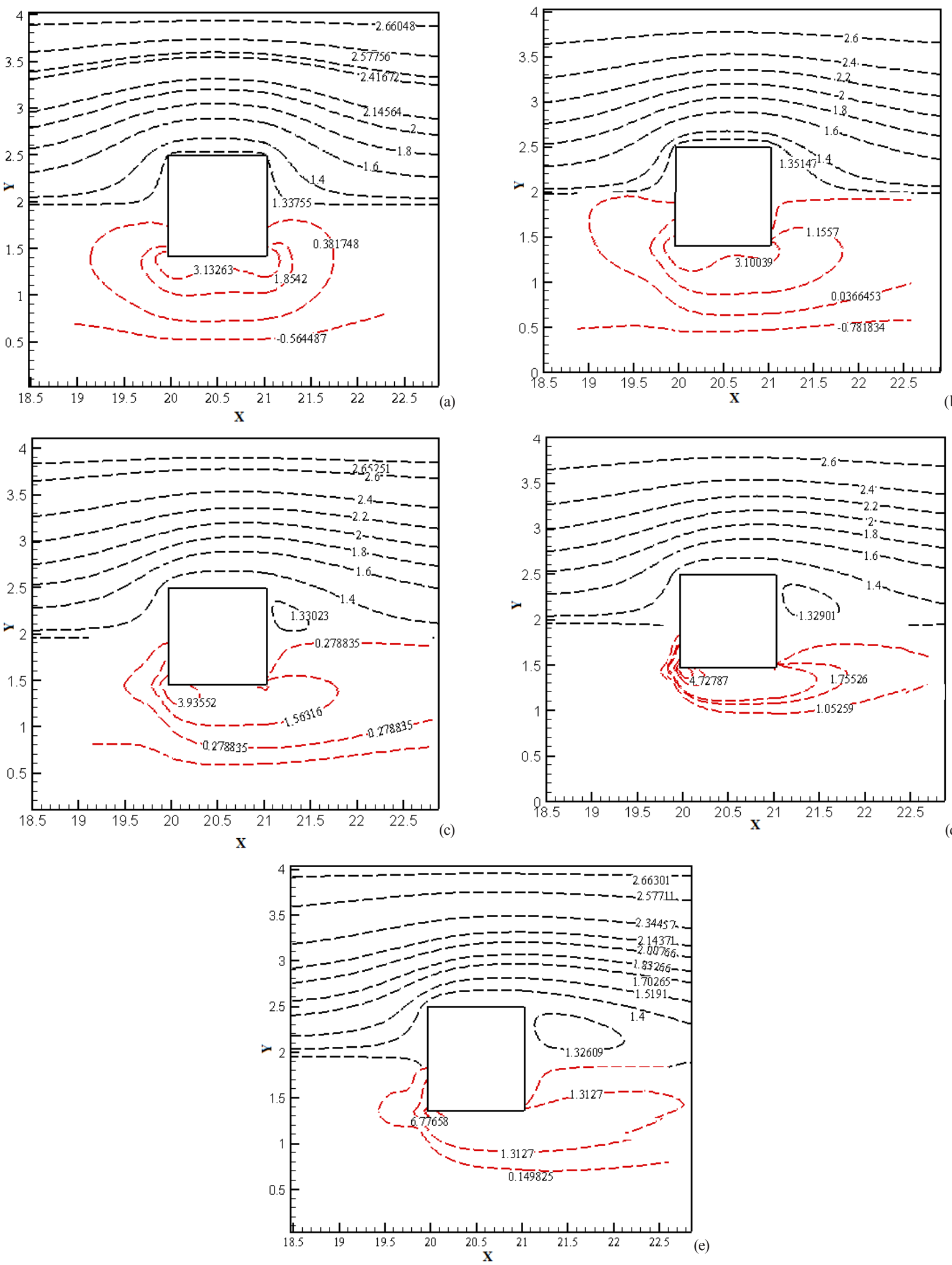

Fig. 4 Streamline and vorticity profiles (upper and lower parts correspond to streamline and vorticity, respectively) for different Reynolds numbers. (a) $R e=0$ (b) $R e=10$ (c) $R e=20$ (d) $R e=30$ (e) $R e=50$ 
Table 1 Intensities of the primary eddies and vorticity and their locations in the wake region

\begin{tabular}{|c|c|c|c|c|}
\hline $\operatorname{Re}$ & $\omega$ & $\psi$ & $x$ & $y$ \\
\hline 0 & -1.8930 & 1.6636 & 22 & 3 \\
\hline 0 & -1.7269 & 1.6874 & 23 & 3 \\
\hline 0 & -1.4988 & 1.7168 & 24 & 3 \\
\hline 0 & -4.3842 & 1.3526 & 22 & 2.5 \\
\hline 0 & -3.0359 & 1.3762 & 23 & 2.5 \\
\hline 0 & -2.2318 & 1.4061 & 24 & 2.5 \\
\hline 10 & -1.9136 & 1.6246 & 22 & 3 \\
\hline 10 & -1.8391 & 1.6379 & 23 & 3 \\
\hline 10 & -1.7330 & 1.6548 & 24 & 3 \\
\hline 10 & -2.9159 & 1.3424 & 22 & 2.5 \\
\hline 10 & -2.3837 & 1.3531 & 23 & 2.5 \\
\hline 10 & -2.0887 & 1.3668 & 24 & 2.5 \\
\hline 20 & -1.9703 & 1.6089 & 22 & 3 \\
\hline 20 & -1.9091 & 1.6177 & 23 & 3 \\
\hline 20 & -1.8289 & 1.6291 & 24 & 3 \\
\hline 20 & -2.6605 & 1.3589 & 22 & 2.5 \\
\hline 20 & -2.2703 & 1.3442 & 23 & 2.5 \\
\hline 20 & -2.0476 & 1.3316 & 24 & 2.5 \\
\hline 30 & -2.0254 & 1.6021 & 22 & 3 \\
\hline 30 & -1.9662 & 1.6084 & 23 & 3 \\
\hline 30 & -1.8932 & 1.6167 & 24 & 3 \\
\hline 30 & -2.3695 & 1.3365 & 22 & 2.5 \\
\hline 30 & -2.1791 & 1.3397 & 23 & 2.5 \\
\hline 30 & -2.0538 & 1.3438 & 24 & 2.5 \\
\hline 50 & -2.0765 & 1.5949 & 22 & 3 \\
\hline 50 & -1.9779 & 1.5985 & 23 & 3 \\
\hline 50 & -1.9034 & 1.6035 & 24 & 3 \\
\hline 50 & -2.1139 & 1.3343 & 22 & 2.5 \\
\hline 50 & -2.0603 & 1.3351 & 23 & 2.5 \\
\hline 50 & -1.9946 & 1.3359 & 24 & 2.5 \\
\hline
\end{tabular}

However, vorticity intensity shows a decreasing trend as compared to upstream locations. It can be resulted from that $v$ velocity component changing with respect to $x$ creates smaller gradients in the wake region for high Re numbers as shown in Fig. 5. Fig. 6 illustrates distribution of the velocity component, $u$ at several positions of the flow field for Re numbers along the center line. At $x=19$ that is the position of near cylinder region, the velocity profiles differs from Poiseuille flow. In the wake region, at $x=22$, maximum velocity point shifts with respect to increasing $R e$. This behavior can be attributed to the vortex formation behind of obstacle. On the other hand, $u$-velocity component changing with $y$ axis has more symmetrical distribution due to the imposed parabolic velocity profile at the

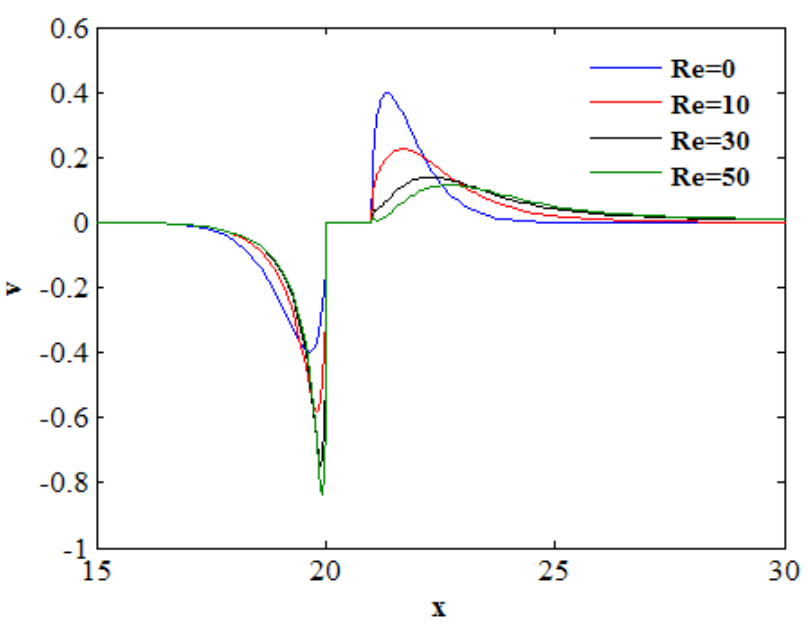

Fig. 5 Vertical velocity (v) along the centerline $y=2$ at $R e=0,10,30,50$

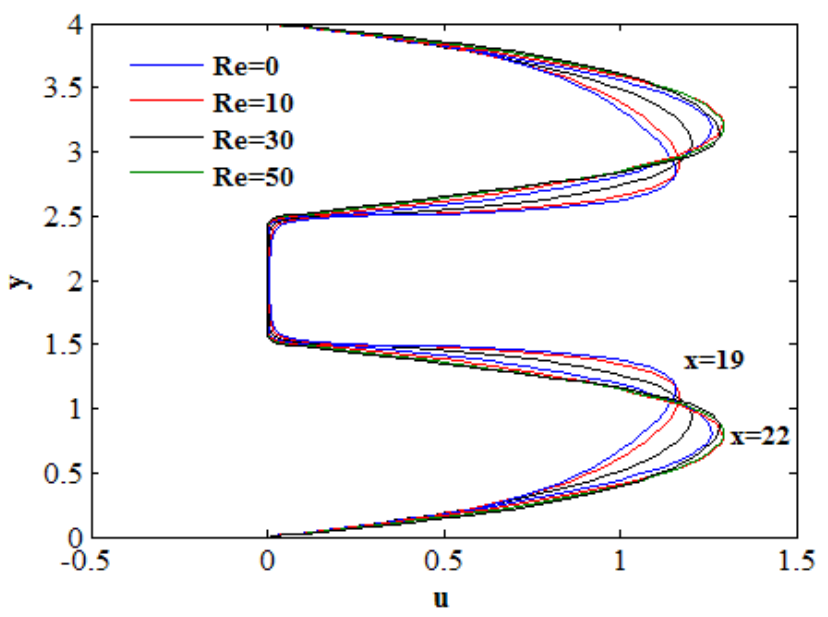

Fig. 6 Velocity component, $u$, profiles for different positions at $x=19$ (near cylinder), $x=22$ (wake region) for $R e=0,10,30,50$ along the centerline $(\mathrm{y}=2)$

inlet of the channel in Fig. 6. It is also nearly independent of Re numbers.

\subsection{Drag coefficient around the obstacle}

Drag coefficient $C d$, is important characteristic quantities for flow around a cylinder. In the region of small Reynolds numbers the drag coefficient changes strongly with $R e$. A comparison of the results of different studies is shown in Table 2 at $0 \leq R e \leq 50$. A Cartesian non-uniformly structured mesh with $372 \times 162$ grid system is used to represent the flow system having a blockage ratio of $B=1 / 8$ and $B=1 / 4$. Drag coefficient is obtained by integrating the shear stress (viscous) and pressure contributions over the square cylinder surfaces denoted as $f$ front, $r$ rear, $t$ top, and $b$ bottom similar to the methodology reported by Dhiman et al. [10]. The relation for $C d$ can be written as: 
$C d=\frac{2}{\operatorname{Re}} \int_{0}^{1}\left[\left(\tau_{x y, t}(x)+\tau_{x y, b}(x)\right) d x\right]+2 \int_{0}^{1}\left[\left(P_{r}(y)-P_{f}(y)\right) d y\right]$

The decreasing trend of $C d$ with increasing $R e$ is captured. The impact of $R e$ on $C d$ becomes more pronounced at low $R e$ region. At very low $R e$ region $(R e<1)$ that strong impact can be deduced through the Stokes drag coefficient as $C d=24 / R e$. The drag results are also listed in Table 2 along with the available values in literature. No data is found in the literature for $B=1 / 4$ for the Newtonian case. Therefore, comparison was only done for $B=1 / 8$. When $B=1 / 8$, the results of this study and those earlier studies compare well with each other. Therefore the methodology followed in this study seems to be accurate to simulate the flow for the Newtonian case. Another interesting result is associated with the effect of the blockage ratio on the $C d$. Due to the Newtonian nature of the flow, the drag originates from the form drag acting on the front and rear surfaces and shear drag on the top and bottom surfaces. When flow gets more restricted due to presence of a larger obstacle, i.e. larger $B$, higher velocities between the channel and the obstacle give rise to higher shear stress and form drag effects, i.e. larger $C d$ values as shown in Table 2 .

\section{Visualization of Flow Field}

PIV is a laser optical measurement technique for analyzing laminar and turbulence flow, microfluidic flow processes. Standard PIV measures two velocity components in a plane using a single camera. The principle behind PIV is to derive velocity vectors from sub-sections of the target area of the particle-seeded flow by measuring the movements of particles between two light pulses [19, 20]:

$v=\frac{\Delta x}{\Delta t}$.

In a standard, two-dimensional system, illumination of the flow field is provided by a narrow sheet of light. The flow is seeded particles, and the images of these particles are recorded by a camera placed at $90^{\circ}$ to the light sheet. Low Reynolds number PIV studies in the literature are scarce due to difficulties encountered to obtain high quality velocity measurements. On the contrary, reports on numerical investigation at low Reynolds number flows are more readily available as mentioned in Introduction part. In this section, we qualitatively describe the sequence of changes that occurs to the flow pattern around a square
Table 2 Comparison of $C d$ in steady flow regime with literature for various $R e$ numbers

\begin{tabular}{cccccc}
\hline Re & $\begin{array}{c}\boldsymbol{B}=\mathbf{1 / 4} \\
\text { (present) }\end{array}$ & $\begin{array}{c}\boldsymbol{B}=\mathbf{1 / 8} \\
\text { (present) }\end{array}$ & $\begin{array}{c}\boldsymbol{B}=\mathbf{1 / 8} \\
{[\mathbf{1 0}]}\end{array}$ & $\begin{array}{c}\boldsymbol{B}=\mathbf{1 / 8} \\
{[\mathbf{9}]}\end{array}$ & $\begin{array}{c}\boldsymbol{B}=\mathbf{1 / 8} \\
{[\mathbf{8}]}\end{array}$ \\
\hline 10 & 8.127 & 3.699 & 3.663 & 3.511 & 3.872 \\
20 & 4.702 & 2.462 & 2.442 & 2.448 & 2.578 \\
30 & 3.556 & 2.214 & - & - & 2.278 \\
40 & 3.236 & 1.826 & 1.852 & 1.864 & 1.925 \\
50 & 2.608 & 1.793 & 1.751 & 1.762 & 1.854 \\
\hline
\end{tabular}

cylinder with blockage ratio of 1/4 with Reynolds number within the range of 1 and 50 using PIV.

A large number of studies have been performed to investigate the Newtonian flow over single square cylinder to analyze turbulence flow characteristics. Okajima et al. [21] carried out an experimental study of flow past a square cylinder as well as rectangular cylinder for $70<R e<20,000$ to determine the vortex shedding frequencies for unsteady flow. Okajima found that the highest Strouhal number $(\mathrm{St})$ was observed when $10^{4}<R e<2 \times 10^{4}$. Van Oudheusden et al. [22] studied the vortex shedding and drag force characteristics in the near wake of a square cylinder placed at various angles $(\theta)$ to the mean flow for Reynolds numbers of 4,000, 10,000, and 20,000 using PIV. They found that the flow separation occurred at both front corners and the shallow recirculation regions were observed above and below the obstacle while the angle is 0 . The separation points move downstream while $\theta>0$. Furthermore, two other large recirculation regions appeared in the wake behind the body in a similar fashion as that for the circular cylinder. According to Berrone and Marro [23] as Re is increased, the upstream-downstream symmetry of the streamlines disappeared and two eddies appeared behind the cylinder. These eddies get bigger with increasing $R e$, the flow becomes unsteady due to wake instability mechanisms and the phenomenon of vortex shedding, known also as von Kármán Street [24]. The literature review indicates the importance of turbulence characteristics for bluff bodies at high Reynolds numbers. These are mainly related to the unsteady flow around the obstacle.

Current PIV measurements were conducted to investigate the impact of square obstacle inside a channel. Particle image velocimetry (PIV) was used to measure the two-dimensional velocity field to reveal streamlines and vorticity patterns in a qualitatively manner. The changes of flow structure of the system due to effect of Re number were compared with computational results obtained at similar conditions. 
PIV visualizations (Dantec Dynamics) were performed at Nanotechnology Engineering Department at Cumhuriyet University, Sivas. The experiments were carried out in a $300 \mathrm{~cm}$ long channel plexiglas of $1 / 2$ in with the inner cross-section of $12 \times 12 \mathrm{~cm}$. The channel and the closed flow loop to circulate clean tap water via a magnetic pump are depicted in Fig. 7. Square obstacle made of plexiglas was installed in the test section as shown in Fig. 8. The dimensions of obstacle were $3 \mathrm{~cm}$ height, $0.5 \mathrm{~cm}$ in thickness, and $12 \mathrm{~cm}$ in width to provide a blockage ratio of $1 / 4 . B$ is the ratio of obstacle diameter over channel diameter. Experiments were done at the Reynolds numbers of 1, 20, 30, 40, 50 where the Reynolds number was defined based on the diameter of the channel and the mean water velocity. To observe two-dimensional flow field of the system, illumination of the flow field was provided by a narrow sheet of light. The flow was seeded by Polyamide particles of size $50 \mu \mathrm{m}$ and the images of these particles were recorded by a camera placed at $90^{\circ}$ to the light sheet depicted in Fig. 7. Solid state Nd: YAG lasers using frequency-doubling crystals to produce light at $532 \mathrm{~nm}$ was used as the light source. 256 pair of images were taken by PIV camera and these images yields velocity vectors by cross-correlating the interrogation region in the first 5 image with the corresponding search region in the second image pair [20,21].

Averages of these images were taken by PIV processor to get flow field. Two dimensional mean velocity vector fields at $R e=50$ is presented in Fig. 9. The plot represents the typical behavior flow past over an obstacle. The flow shows that the flow is affected by the obstacle mainly in the surrounding region with the flow separation off the top and bottom edges of the obstacle. Fig. 9 also exhibits strong recirculation region at downstream of the obstacle. Stagnation areas are observed near the obstacle wall.

Vortices induced by the obstacle enhance mixing in the recirculation zone. Subsequent plots in Figs. 10(b) and 10 (e) give the vortex patterns. These locations are the extent of the flow within the given field of view from the region immediately downstream of the square obstacle including the recirculation zone up to the region where the flow reattaches itself. Comparing the flow patterns for both results, symmetrical vortex region can be identified similar to the numerical results in Fig. 4. At $R e=20$, small vortexes start to occur near attachment of the wake region as shown in Fig. 10(b). The wake area or recirculation region also increases as the effect of inertial forces increases when $R e$ is increased from $R e=30$ to $R e=50$.

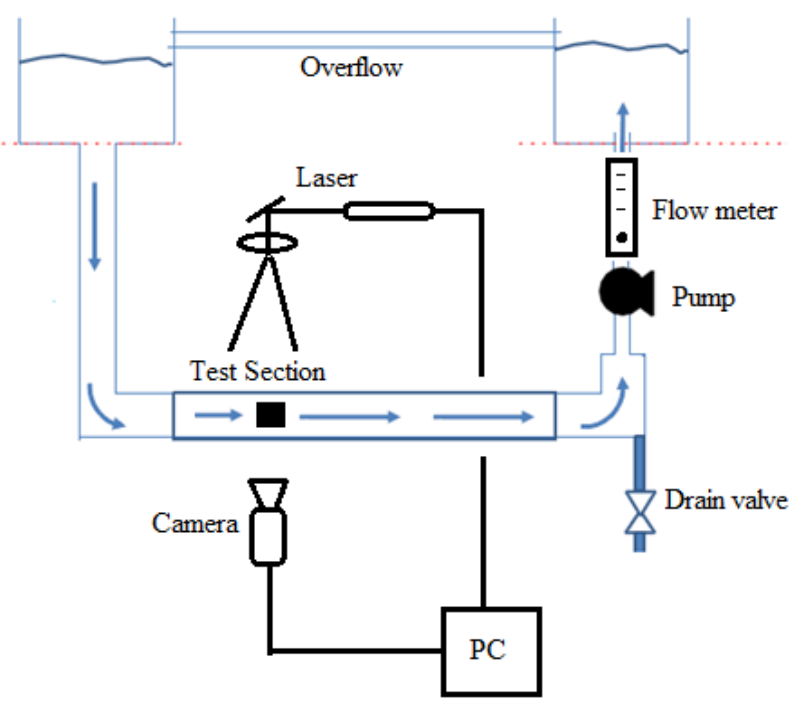

Fig. 7 Experimental flow loop set-up

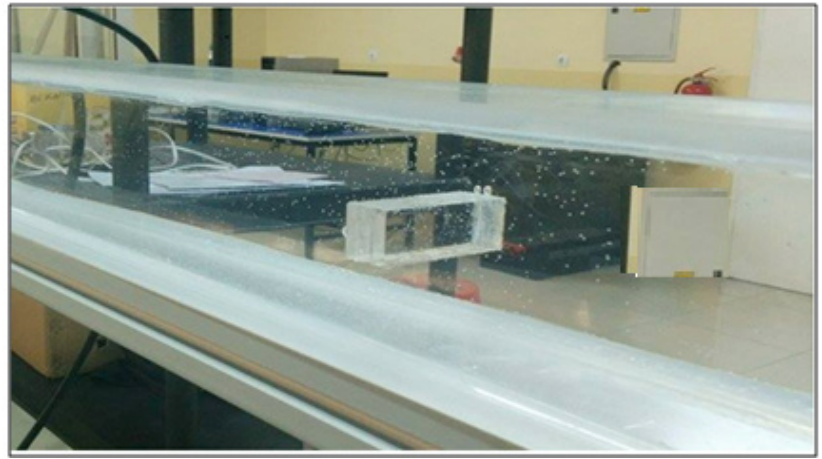

Fig. 8 Illustration of square obstacle inside experimental set-up

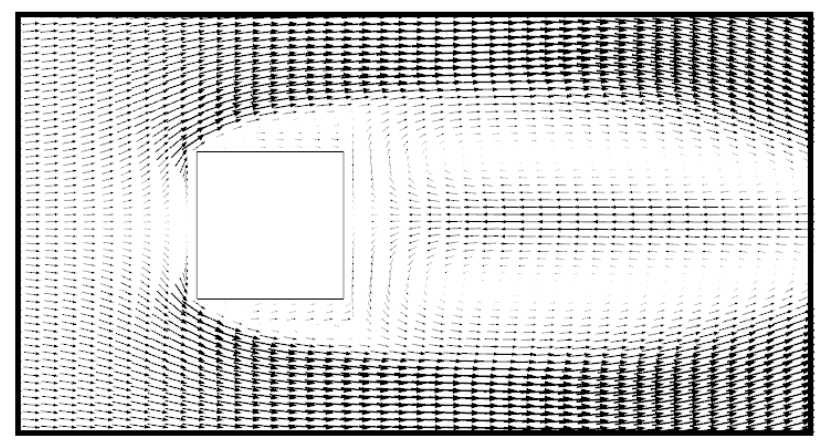

Fig. 9 Experimental velocity vector field at $R e=50$

With an increase in $R e$ number, symmetrical vortexes become more discernible due to the increased accuracy of PIV at higher velocities. Large part of the flow domain is affected by the obstacle and larger vortex appeared behind the obstacle as shown in Figs. 10(d)-(e) compared to Figs. 10(b)-(c) when the Reynolds number increased.

Vorticity field is calculated based on the velocity field shown in Fig. 10. At the edges of the obstacle where 



(e)

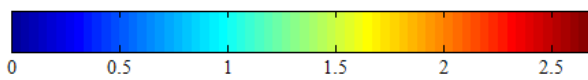

Fig. 10 Experimental streamline profiles around the square cylinder for different Reynolds numbers by PIV (a) $R e=1$ (b) $R e=20$ (c) $R e=30$ (d) $R e=40$ (e) $R e=50$. Contour levels are shown from 0 to 2.5 with the increment of 0.5 .

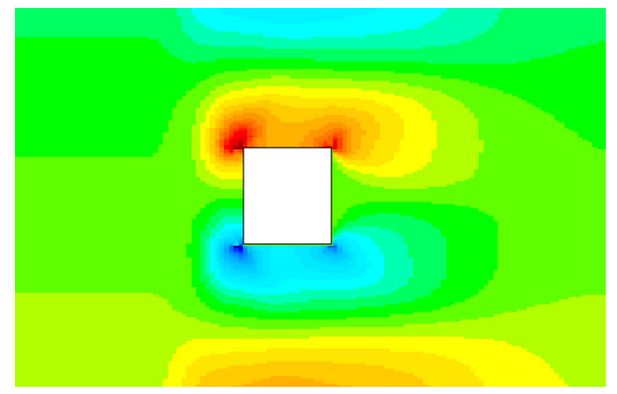

(a)

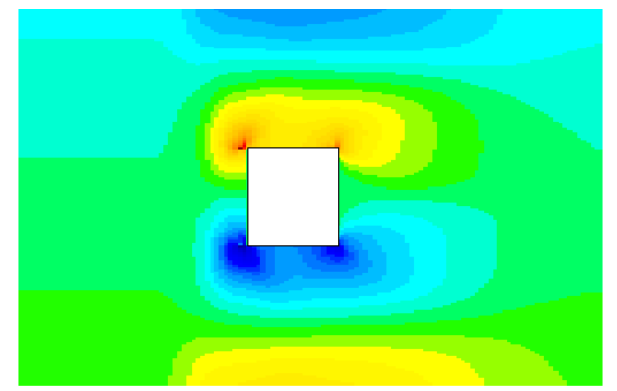

(b)

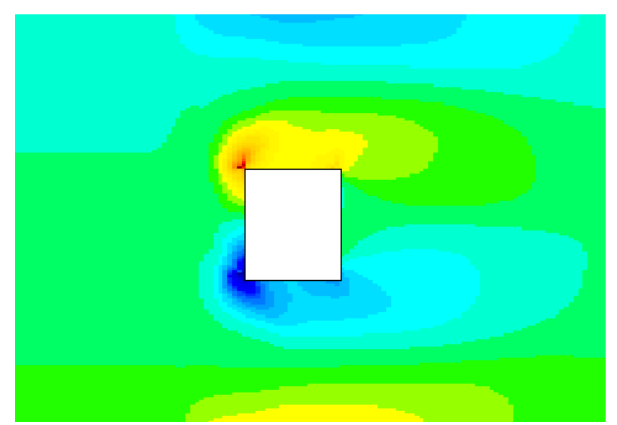

(c)

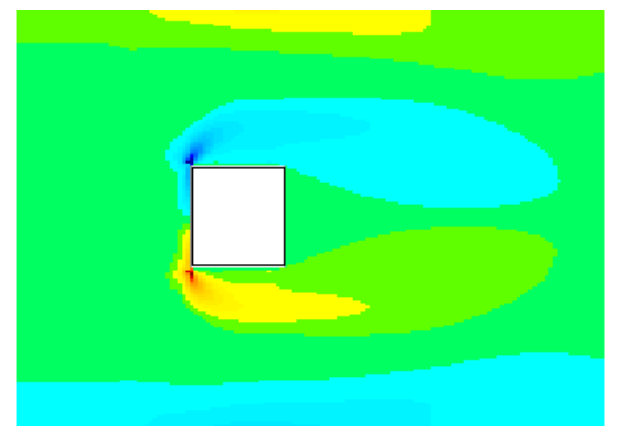

(d)

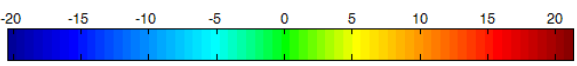

Fig. 11 Experimental vorticity profiles around the square cylinder for different Reynolds numbers by PIV (a) $R e=1$ (b) $R e=10$ (c) $R e=30$ (d) $R e=50$. Contour levels are shown from -20 to 20 with increment of 5 . 
boundary conditions of the system changes suddenly, highest intensity of the vorticity is obtained in the normal direction to the flow. But in the region between obstacle and the channel wall there is high shearing of the fluid and the vorticity magnitude is lower in this region as shown in Fig. 11. In order to study influence of Re on the vorticity dynamics of Newtonian flow around the obstacle, contour maps of the vorticity field of flow for different Reynolds numbers are presented in the following plots in Figs. 11(a)-(d). There are qualitative similarities between numerical and experimental results. For all Reynolds numbers, symmetrical vorticity distribution is attained in Figs. 11(a)-(d). Vorticity is almost uniform and its intensity remains the same at the corner of the obstacle depicted in Figs. 11(a) and 11(d).

Increase of flow inertia leads to increase in the intensity of the vorticity contours as shown in Figs. 11(c) and 11(d). Contour layers at the edges of the obstacle are more distinguishable, a behavior similar to the numerical predictions. As Reynolds number increases, vorticity pattern disperses symmetrically in the flow direction as shown in Fig. 4. Vortex area is also found to be higher as Reynolds number gets larger.

\section{Conclusions}

In this study numerical and experimental studies are carried out for steady laminar flows of both Newtonian fluid around the confined square cylinder to reach physical mechanism of flow behavior around the square obstacle. Particle image velocimetry (PIV) was also used to obtain the two-dimensional velocity field. The results obtained in this study allow one to draw the following conclusions:

- Symmetrical vortex structure are found to be higher with an increase in the Reynolds number for

\section{References}

[1] Nitin, S., Chhabra, R. P. "Non-isothermal flow of a power law fluid past a rectangular obstacle (of aspect ratio $1 \times 2$ ) in a channel: Drag and heat transfer", International Journal of Engineering Science, 43(8-9), pp. 707-720, 2005.

https://doi.org/10.1016/j.ijengsci.2004.12.015

[2] Zdravkovich, M. M. "Flow around Circular Cylinders; Volume 1. Fundamentals." 1st ed., Oxford University Press, Oxford, England, 1997.

http://doi.org/10.1017/S0022112097227291

[3] Rashidi, S., Esfahani, J. A., Ellahi, R. "Convective Heat Transfer and Particle Motion in an Obstructed Duct with Two Side by Side Obstacles by Means of DPM Model", Applied Sciences, 7(4), pp. 431-444, 2017.

https://doi.org/10.3390/app7040431
Newtonian flow around the obstacle at $R e>20$ as in Figs. 4(c)-(e) and Figs. 10(c)-(e).

- Highest intensity of vorticity is obtained in the normal direction to the flow. Symmetrical vortex structures at the wake region is observed when $R e$ increased as in Figs. 4 and 10.

- Numerical simulations is confirmed by the experimental visualization method. Particle image velocimetry (PIV) was used to measure the two-dimensional velocity field to reveal streamlines and vorticity patterns in a qualitatively manner. The changes of flow structure of the system due to effect of Re number were compared with computational results obtained at similar conditions as in Figs. 10 and 11.

- The effect of inertia on vorticity distribution around the obstacle is analyzed and the results getting of two methods show that increasing the Reynolds number leads to increase of vortex area and intensity.

- The impact of Re number on $C d$ becomes more pronounced at low Re region as listed in Table 2. As inertial effects increase, the contributions of the viscous effect and pressure effect to the total drag coefficient decrease for Newtonian flow.

$\begin{array}{cl}\text { Nomenclature } \\ R e & \text { Reynolds number } \\ B & \text { Blockage ratio } \\ \tau_{x y} & \text { Shear stress } \\ P & \text { Pressure } \\ u & \text { Velocity } \\ C d & \text { Drag coefficient } \\ n & \text { Power law index } \\ \omega & \text { Intensity of the primary vortex } \\ \psi & \text { Stream function }\end{array}$

[4] Shirvan, K. M., Ellahi, R., Mamourian, M., Moghiman, M. "Effect of Wavy Surface Characteristics on Heat Transfer in a Wavy Square Cavity Filled with Nanofluid", International Journal of Heat and Mass Transfer, 107, pp. 1110-1118, 2017. http://doi.org/10.1016/j.ijheatmasstransfer.2016.11.022

[5] Rashidi, S., Akar, S., Bovand, M., Ellahi, R. "Volume of fluid model to simulate the nanofluid flow and entropy generation in a single slope solar still", Renewable Energy, 115, pp. 400-410, 2018. http://doi.org/10.1016/j.renene.2017.08.059

[6] Shirvan, K. M., Mamourian, M., Mirzakhanlari, S., Ellahi, R. "Numerical Investigation of Heat Exchanger Effectiveness in a Double Pipe Heat Exchanger Filled With Nanofluid: A Sensitivity Analysis by Response Surface Methodology", Power Technology, 313, pp. 99-111, 2017. https://doi.org/10.1016/j.powtec.2017.02.065(.) 
[7] Ellahi, R., Fetecau, C., Sheikholeslami, M. "Recent Advances in the Application of Differential Equations in Mechanical Engineering Problems", Mathematical Problems in Engineering, 2018(1), pp. 1-3, 2018.

https://doi.org/10.1155/2018/1584920

[8] Breuer, M., Bernsdorf, J., Zeiser, T., Durst, F. "Accurate computations of the laminar flow past a square cylinder based on two different methods: lattice-Boltzmann and finite-volume", International Journal of Heat and Fluid Flow, 21(2), pp. 186-196, 2000. https://doi.org/10.1016/S0142-727X(99)00081-8

[9] Gupta, A. K., Sharma, A., Chhabra, R. P., Eswaran, V. "TwoDimensional Steady Flow of a Power-Law Fluid Past a Square Cylinder in a Plane Channel: Momentum and Heat-Transfer Characteristics", Industrial and Engineering Chemistry Research, 42(22), pp. 5674-5686, 2003.

https://doi.org/10.1021/ie030368f

[10] Dhiman, A. K., Chhabra, R. P., Eswaran, V. "Steady flow across a confined square cylinder: Effects of power-law index and blockage ratio", Journal of Non-Newtonian Fluid Mechanics, 148(1-3), pp. 141-150, 2008.

https://doi.org/10.1016/j.jnnfm.2007.04.010

[11] Adrian, R. J. "Twenty years of particle image velocimetry", Experiments in Fluids, 39(2), pp. 159-169, 2005.

https://doi.org/10.1007/s00348-005-0991-7

[12] Bird, R. B., Stewart, W. E., Lightfoot, E. N. "Transport phenonema", 1st ed., John Wiley and Sons Inc., New York, USA, 1960. https://doi.org/10.1002/aic.690070245

[13] Versteeg, H. K., Malalasekera, W. "An Introduction to Computational Fluid Dynamics: The Finite Volume Method", 1st ed., Pearson Prentice Hall, London, England, 1995.

[14] Perić, M., Kessler, R., Scheuerer, G. "Comparison of finite-volume numerical methods with staggered and colocated grids", Computers \& Fluids, 16(4), pp. 389-403, 1988. https://doi.org/10.1016/0045-7930(88)90024-2

[15] Alves, M. A., Oliveira, P. J., Pinho, F. T. "A convergent and universally bounded interpolation scheme for the treatment of advection", International Journal for Numerical Methods in Fluids, 41(1), pp. 47-75, 2003. https://doi.org/10.1002/fld.428

[16] Rubin, S. G., Khosla, P. K. "Polynomial interpolation methods for viscous flow calculations", Journal of Computational Physics, 24(3), pp. 217-244, 1977. https://doi.org/10.1016/0021-9991(77)90036-5
[17] Hayase, T, Humphrey, J. A. C, Greif, R. "A consistently formulated QUICK scheme for fast and stable convergence using finite-volume iterative calculation procedures", Journal of Computational Physics, 98(1), pp. 108-118, 1992. https://doi.org/10.1016/0021-9991(92)90177-Z

[18] Yapici, K. "A comparison study on high-order bounded schemes: Flow of PTT-linear fluid in a lid-driven square cavity", KoreaAustralia Rheology Journal, 24(1), pp. 11-21, 2012. https://doi.org/10.1007/s13367-012-0002-5

[19] Keane, R. D., Adrian, R. J. "Theory of cross-correlation analysis of PIV images", Applied Scientific Research, 49(3), pp. 191-215, 1992. https://doi.org/10.1007/BF00384623

[20] Westerweel, J., Elsinga, G. E., Adrian, R. J. "Particle Image Velocimetry for Complex and Turbulent Flows", Annual Review of Fluid Mechanics, 45(1), pp. 409-436, 2013. https://doi.org/10.1146/annurev-fluid-120710-101204

[21] Okajima, A., Ueno, H., Sakai, H. "Numerical Simulation of Laminar and Turbulent Flows around Rectangular Cylinders", International Journal for Numerical Methods in Fluids, 15(9), pp. 999-1012, 1992. https://doi.org/10.1002/fld.1650150906

[22] van Oudheusden, B. W., Scarano, F., van Hinsberg, N. P., Watt, D. W. "Phase-resolved characterization of vortex shedding in the near wake of a square-section cylinder at incidence", Experiments in Fluids, 39(1), pp. 86-98, 2005. https://doi.org/10.1007/s00348-005-0985-5

[23] Berrone, S., Marro, M. "Space-time adaptive simulations for unsteady Navier-Stokes problems", Computers \& Fluids, 38(6), pp. 1132-1144, 2009. https://doi.org/10.1016/j.compfluid.2008.11.004

[24] Zandbergen, P. J., Dijkstra, D. "Von Karman Swirling Flows", Annual Review of Fluid Mechanics, 19(1), pp. 465-491, 1987. https://doi.org/10.1146/annurev.fl.19.010187.002341

[25] Sharma, A., Eswaran, V. "Effect of Channel Confinement on the Two-Dimensional Laminar Flow and Heat Transfer across a Square Cylinder", Numerical Heat Transfer, Part A: Applications, 47(1), pp. 79-107, 2004. https://doi.org/10.1080/10407780490520760 